\title{
Spontaneous synthesis of a homogeneous thermoresponsive polymer network composed of polymers with a narrow molecular weight distribution
}

\author{
Yuto Jochi ${ }^{1}$, Takahiro Seki ${ }^{1}$, Takamasa Soejima ${ }^{1}$, Kotaro Satoh ${ }^{1}$, Masami Kamigaito ${ }^{1}$ and Yukikazu Takeoka ${ }^{1}$
}

\begin{abstract}
We have developed a method to obtain a polymer gel consisting of a relatively homogeneous network structure composed of polymers with a narrow molecular weight simply by mixing the necessary compounds. In this method, a radical chain polymerization reaction with a fast reaction rate is combined with a radical addition cross-linking reaction that has a sufficiently slow reaction rate compared with the polymerization reaction. For the polymerization reaction, a multifunctional initiator with a strictly controlled number of polymerization initiation sites was used to prepare a star polymer with a chain number and length determined by living radical polymerization. In the cross-linking reaction, a multifunctional terminating agent was used to prepare a star polymer from a polymer with a narrow molecular weight via living radical polymerization followed by coupling with multiple polymer chains using a termination reaction. As a result, two kinds of reactions with greatly different kinetics occurred sequentially only when all of the compounds were mixed and allowed to stand at a constant temperature, and a relatively homogeneous network structure comprising cross-linked polymers with a low molecular weight distribution was inherently constructed.
\end{abstract}

\section{Introduction}

Polymer gels consist of a cross-linked three-dimensional polymer network swollen with excess solvent ${ }^{1,2}$. Fundamental studies using polymer gels have been undertaken in various disciplines, such as chemistry, physics, biology, and pharmacology ${ }^{3-6}$. Applied research utilizing polymer gels has also been extended to a wide variety of fields, including medicine, civil engineering, architecture, and space exploration ${ }^{7,8}$. In particular, there are two reasons why polymer gels are used in a wide range of basic and applied fields. First, anyone can obtain polymer gels that exhibit similar properties simply by dissolving the necessary monomers and cross-linking agent in a solvent and then degassing and introducing the initiator. Second, the

Correspondence: Kotaro Satoh (satoh@chembio.nagoya-u.ac.jp) or Yukikazu Takeoka (ytakeoka@chembio.nagoya-u.ac.jp)

${ }^{1}$ Department of Molecular and Macromolecular Chemistry, Nagoya University, Furo-cho, Chikusa-ku, Nagoya 464-8603, Japan polymer gels obtained by this simple method feature various functional properties, such as molecular sieving abilities, super absorbency, sustained release capability, and lens function. Briefly, because everyone can easily prepare polymer gels with various possibilities, polymer gels are of interest as research subjects, and their applied research has been widely reported. However, the polymer networks of polymer gels prepared by conventional radical polymerization have nonhomogeneous network structures composed of polymer chains of different molecular weights (Scheme S1) ${ }^{9-11}$. Therefore, conventional polymer networks may not fully exhibit the various functions of polymer gels, such as molecular sieving ability, mechanical strength, transparency, solvent retention capacity, stimuli responsiveness, and biocompatibility. If a polymer gel can be prepared from a polymer network composed of monodisperse polymers, a gel with improved functionality can be obtained.

\section{(c) The Author(s) 2018}

(c) Open Access This article is licensed under a Creative Commons Attribution 4.0 International License, which permits use, sharing, adaptation, distribution and reproduction cc) in any medium or format, as long as you give appropriate credit to the original author(s) and the source, provide a link to the Creative Commons license, and indicate if changes were made. The images or other third party material in this article are included in the article's Creative Commons license, unless indicated otherwise in a credit line to the material. If material is not included in the article's Creative Commons license and your intended use is not permitted by statutory regulation or exceeds the permitted use, you will need to obtain permission directly from the copyright holder. To view a copy of this license, visit http://creativecommons.org/licenses/by/4.0/. 


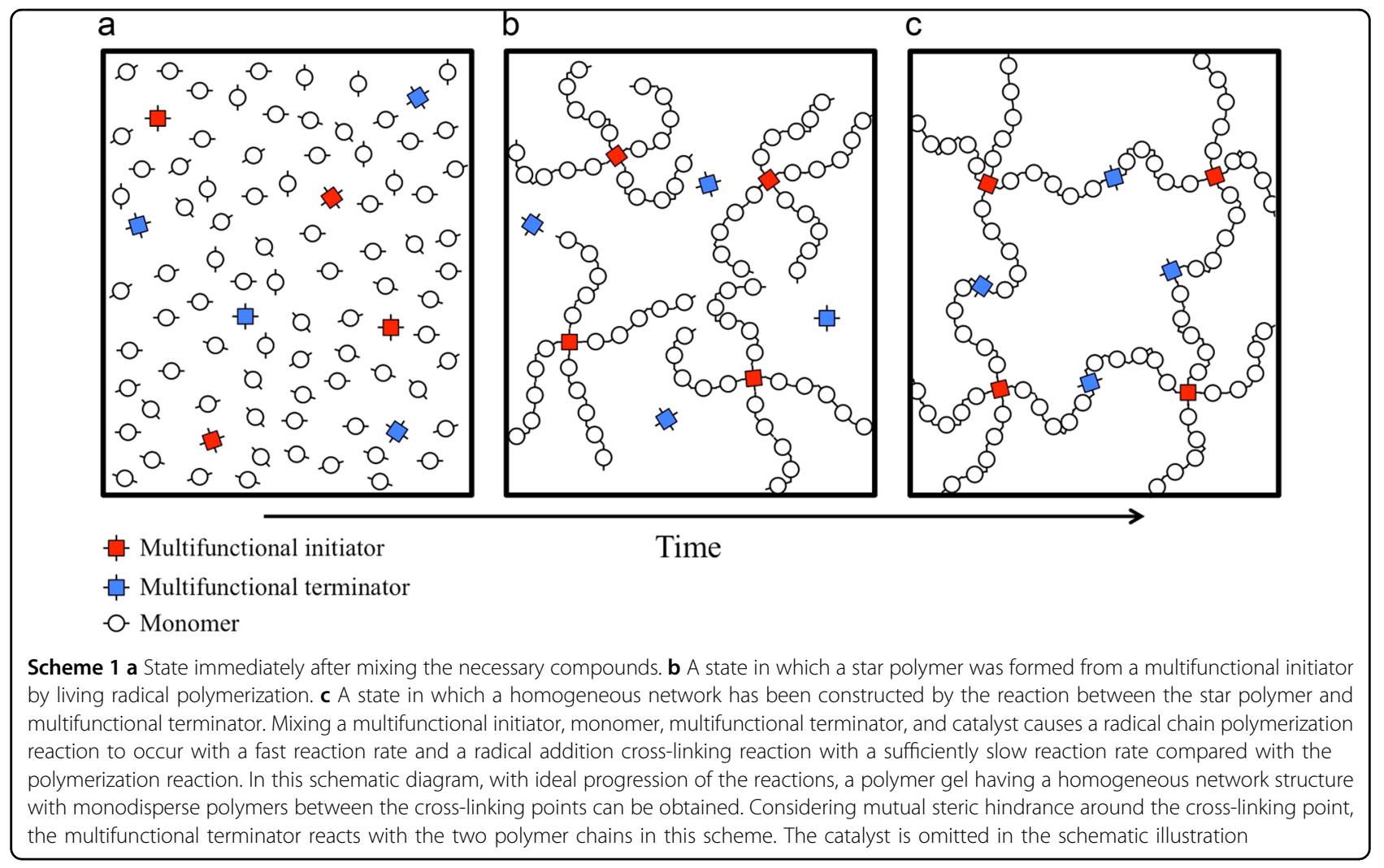

Previously, the construction of a polymer gel having a network structure composed of monodisperse polymers was attempted by the synthesis of linear, high-molecularweight, reactive monodisperse polymers followed by multifunctional cross-linking ${ }^{12-14}$. In addition, other twostep preparation methods have been reported, such as a method of reacting polymer precursors composing monodisperse polymers with four terminal reactive groups and then cross-linking them ${ }^{15-17}$. The polymer gels obtained by these methods have good mechanical properties and optical transparency. If we can find a simpler synthetic method to obtain polymer gels with network structures composed of monodisperse polymers using various other kinds of polymers, then polymer gels with improved functionalities compared to those of the gels studied previously may be achieved. Furthermore, studying the applications of polymer gels composed of monodisperse polymers to a wide range of fields will lead to new findings.

In this work, we sought to develop a method that can inherently synthesize a network structure composed of monodisperse polymers simply by mixing the necessary compounds (Scheme 1). To realize this goal, a polymerization reaction with a fast reaction rate and a crosslinking reaction with a sufficiently slow reaction rate compared with the polymerization reaction, both of which are driven by the same catalyst, were combined. With the recent development of living radical polymerization, many reactions capable of producing various kinds of monodisperse polymers in a short time have been discovered $^{12,18-20}$. For example, in a polymerization reaction of $\mathrm{N}$-isopropylacrylamide (NIPA) using a halogenated organic compound as an initiator and a copper catalyst, a polymer with a desired molecular weight and narrow molecular weight distribution (MWD) can be obtained in almost $100 \%$ yield within a few hours ${ }^{21-23}$. On the other hand, the same copper catalyst used in this polymerization can also catalyze a reaction in which a halogenated organic compound is added to an allyl group $^{24,25}$. This catalyst enables simultaneous chain growth and sequential polymerization by combining sequential polymerization using this addition reaction and living radical polymerization. Here, since the rate of the addition reaction to the allyl group is much slower than that of the polymerization reaction of NIPA, when the initial concentration of NIPA is increased, polymers of narrow molecular weight are first formed by living radical polymerization and then become periodic multiblock copolymers via the addition reaction. We thought that the reaction between halogen-terminated polymers, which are prepared by the living radical polymerization method mentioned above, and a compound with multiple allyl groups could be utilized as a slow cross-linking reaction. 
From this perspective, we prepared a polymer network composed of polymers of a narrow MWD by combining the multifunctional initiator method and a multifunctional terminator method. In the multifunctional terminator method, polymers of relatively narrow molecular weight are prepared beforehand by living radical polymerization and then coupled with multiple polymer chains via a termination reaction to prepare a star polymer. After the necessary compounds were mixed, two reactions with different reaction rates occurred sequentially in an attempt to synthesize a polymer gel with a relatively homogeneous network structure from polymers of a narrow MWD. As a result, we elucidated a possible method of inherently constructing a polymer gel composed of a relatively homogeneous network structure in which various types of macromolecules of narrow molecular weight are cross-linked.

\section{Results and discussion}

\section{Synthesis of a four-branched star polymer by the} multifunctional initiator method

In the polymerization of vinyl monomers using a copper catalyst and a halogenated organic compound as an initiator in a mixed solvent system of dimethylformamide (DMF) and water, a disproportionation reaction of copper occurs, initiating single-electron transfer living radical polymerization $^{26}$. As a result, a polymer with the desired monodisperse polymers can be obtained in a short time and in high yield. Using single-electron transfer living radical polymerization of a multifunctional initiator, namely, the four-branched halogenated organic compound pentaerythritol tetra(2-chloropropionate) (PETCP), enabled the synthesis of a star polymer composed of four monodisperse polymers.

First, to investigate the influence of the solvent composition on the polymerization reaction, PETCP was employed as the initiator in a mixed solvent system of DMF/water at various compositions in which NIPA was used as the vinyl monomer to generate a thermoresponsive polymer. The reaction was thus performed in the DMF/water mixed solvent system with different DMF compositions $(25,50$, or 75 vol\% DMF). The NIPA polymerization was performed at a reaction temperature of $4{ }^{\circ} \mathrm{C}$ and a total monomer concentration of $2 \mathrm{M}$ using $1 / 2000$ equivalents of PETCP (relative to NIPA). Figure 1a shows the progress of the polymerization reaction in which PETCP, NIPA, and $\mathrm{CuCl}$ as the catalyst and $\mathrm{Me}_{6}$ TREN as its ligand were dissolved in the mixed solvent system of various compositions of DMF/water under an argon atmosphere. Immediately after all of the compounds were mixed, the polymerization of NIPA proceeded, and when the composition of DMF was 25\%, almost all of the NIPA was consumed in approximately 2 h. When the composition of DMF was $50 \%$, the reaction rate slightly decreased compared with that with $25 \%$ DMF, but over $95 \%$ of the monomer was still consumed in approximately $4 \mathrm{~h}$. On the other hand, when the composition of DMF was $75 \%$, the progress of the polymerization became much slower, and only approximately $67 \%$ of the NIPA was polymerized even after $24 \mathrm{~h}$.

The results of plotting $\ln [M]_{0} /[M]$ versus the reaction time are shown in Fig. 1b. Note that $[\mathrm{M}]_{0}$ is the initial concentration of the monomer, and $[\mathrm{M}]$ is the concentration of the monomer at a given time. In all systems, the first-order plot showed a linear relationship. The reaction rate constant $k$ was obtained from the slope of this straight line, and as the DMF composition ratio in the DMF/water mixed solvent system increased, $k$ decreased. These results confirmed that a higher water composition ratio in the mixed solvent system resulted in a higher polymerization rate.

Plots of the number average molecular weight $\left(M_{\mathrm{n}}\right)$ and MWD $\left(M_{\mathrm{w}} / M_{\mathrm{n}}\right)$ obtained by size-exclusion chromatography (SEC) against the percent conversion are shown in Fig. 1c, d, respectively. It was confirmed that the conversion and number average molecular weight were well controlled and in a proportional relationship under all three conditions. In contrast, a large difference was found between the conversion and the MWD. During the initial stage of polymerization (reaction extent of $25 \%$ or less), a larger DMF composition ratio resulted in a smaller MWD of the obtained polymer. Comparing the polymerizations performed using 25 vol\% of DMF and 50 vol\% of DMF revealed that narrow MWD polymers can be obtained initially in the latter case. On the other hand, for the polymerization carried out under the condition of $75 \mathrm{vol} \%$ DMF, the chain elongation step hardly proceeded, and the MWD also increased after the reaction extent reached $60 \%$ in the latter half of the polymerization. In fact, it has been reported that the same trend was observed in the synthesis of linear PNIPA, which is consistent with the results in this study ${ }^{22}$. These results indicate that a DMF/ water mixed solvent system with 25 or $50 \%$ DMF efficiently accelerated the polymerization reaction of NIPA and provided polymers with controlled molecular weight.

\section{Influence of solvent composition and temperature on the swelling of a poly( $\mathrm{N}$-isopropylacrylamide) (PNIPA) gel}

PNIPA and PNIPA gels are known to exhibit concave solubility or degree of swelling curves in a mixture of an organic solvent and water ${ }^{27-30}$. Depending on the composition of the mixed solvent, even when the monomer is dissolved in the solvent system, the resultant polymer may not stay dissolved. That is, when a mixed solvent is used for the gelation of NIPA, the PNIPA gel adopts a collapsed state under certain solvent compositions. When a PNIPA gel is synthesized in these solvent systems, the polymer aggregates and precipitates, preventing the 
(a)

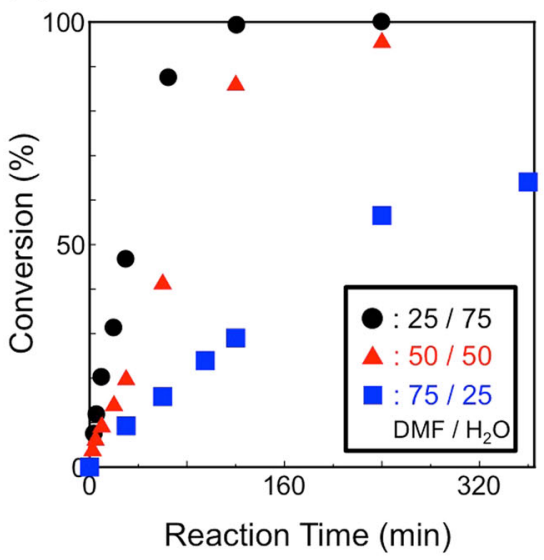

(c)

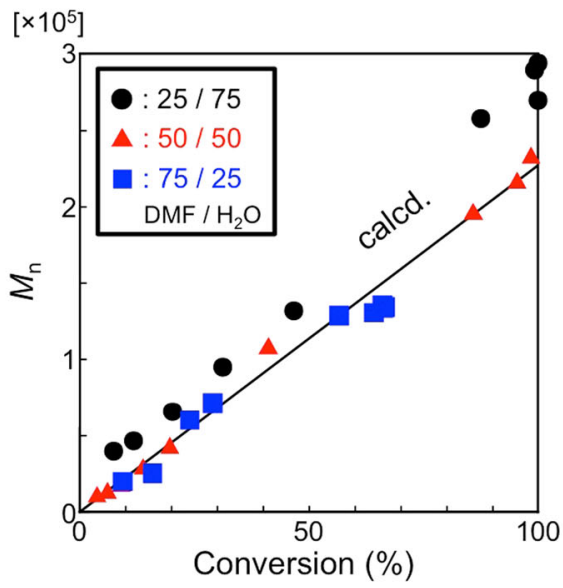

(b)

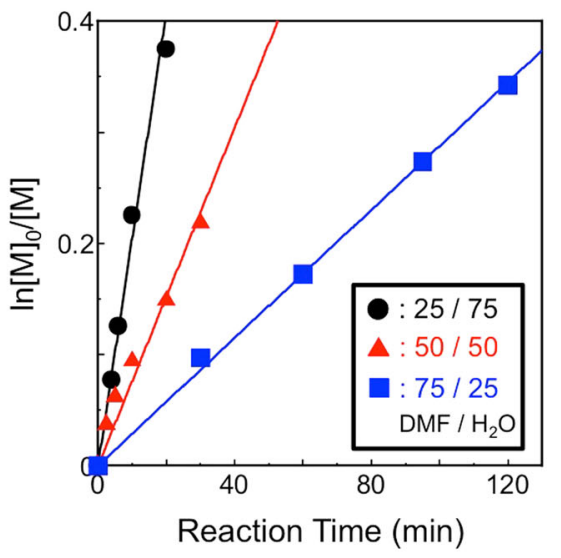

(d)

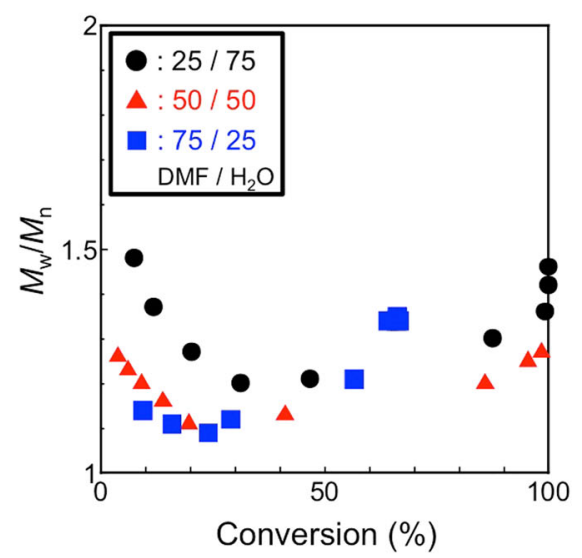

Fig. 1 Results of the living polymerization of NIPA using a mixed solvent system of different ratios of DMF and water. a Diagram plotting the monomer-to-polymer conversion versus reaction time. $\mathbf{b}$ Based on the result of $\mathbf{a}$, a diagram considering polymerization as the primary reaction. c Diagram showing the relationship between the number of average molecular weight of the obtained polymer and the conversion. $\mathbf{d}$ Diagram showing the relationship between the MWD and the conversion

formation of a polymer gel having a homogeneous network structure composed of polymers with a desired molecular weight ${ }^{31}$. To prepare a polymer gel with a homogeneous network structure, it is preferable that the solvent used be a sufficiently good solvent for the constituent polymer, which ensures that the polymer gel is sufficiently swollen.

Therefore, a cylindrical PNIPA gel prepared by a conventional radical polymerization method using methylenebisacrylamide (MBAA), which is a commonly used cross-linking agent, was used to investigate the composition dependence of the degree of swelling of the PNIPA gel in the DMF/water mixed solvent system. This PNIPA gel was prepared using water as a solvent at $4{ }^{\circ} \mathrm{C}$ because, under these conditions, it behaves as a sufficiently good solvent $^{32}$. Additionally, as the quality of the solvent for the polymerization can change depending on both the temperature and composition of the solvent, the effect of temperature on the degree of swelling of the PNIPA gel was also investigated. Figure 2 shows the degree of swelling of the PNIPA gel measured at different temperatures in a DMF/water mixed solvent system of various compositions. The degree of swelling $L / L_{0}$ is obtained, where $L_{0}$ is the length of the polymer gel at the time of preparation and $L$ is the length of the polymer gel under a given condition. Under conditions with $50 \%$ or less DMF in the mixed solvent system and temperatures of $35^{\circ} \mathrm{C}$ or less, as the amount of water increased, the PNIPA gel swelled greatly but shrank with increasing temperature ${ }^{30}$. In contrast, the PNIPA gel shrank when the amount of water increased to $40^{\circ} \mathrm{C}$ or higher. When $50 \%$ or more DMF was used, the temperature dependence was small, 


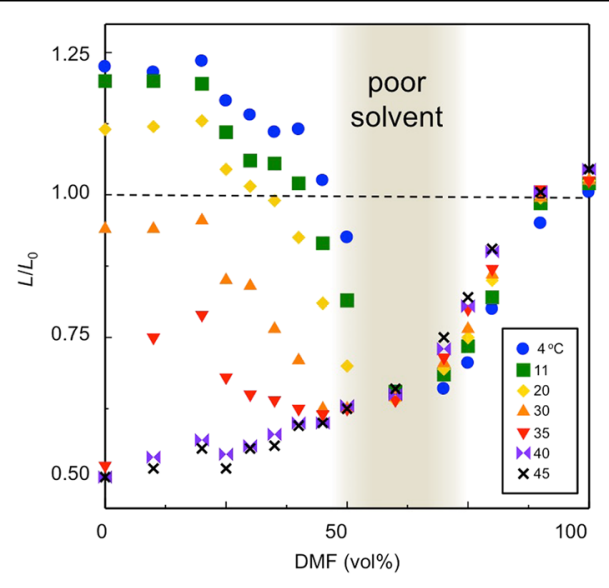

Fig. 2 Degree of swelling of the polymer gel composed of NIPA and MBAA prepared by conventional radical polymerization as measured by changing the solvent composition and temperature

but swelling still occurred when the amount of DMF was large. That is, it was found that, at $35^{\circ} \mathrm{C}$ or less, the PNIPA gel in the DMF/water mixed solvent system exhibits a concave degree of swelling curve compared with the gels in each of the pure solvents. When the gelation of NIPA is carried out under the condition that the value of $L / L_{0}$ is 1 or less, aggregation can be envisaged as the polymer network is formed, and a polymer gel with coarse density in the network structure can be obtained. Therefore, it is considered desirable to synthesize a polymer gel at a solvent composition and temperature such that the value of $L / L_{0}$ becomes 1 or more. Based on the polymerization conditions of NIPA and the swelling behavior of the PNIPA gel described above, it is understood that a PNIPA gel should be prepared at a low temperature of $20^{\circ} \mathrm{C}$ or less using a mixed solvent system with less than 50\% DMF.

\section{Preparation of homogeneous PNIPA gels}

We have worked on the preparation of PNIPA gels using mixed solvents of various DMF compositions. In addition to PETCP, NIPA, $\mathrm{CuCl}$, and $\mathrm{Me}_{6}$ TREN, 1,3,4,6tetraallylglycoluril (TA-G), a four-branched allyl compound, was also dissolved in the DMF/water mixed solvent system of various compositions under an argon atmosphere to prepare the pre-gel solutions, and the time course of the reaction was observed. In all of the systems, until the progress of the polymerization of NIPA initiated by PETCP was completed (i.e., at $5 \mathrm{~h}$ ), the viscosity increased but the reaction mixture remained in solution state. However, in the system with a DMF composition of $25 \%$, gelation occurred after $6 \mathrm{~h}$ (Fig. 3). In other words, in this reaction system, following the completion of the polymerization reaction of NIPA initiated by PETCP, the halogen terminal group on each polymer chain underwent the addition reaction with TA-G as a multifunctional terminator, thereby affording a polymer gel. In this system, 5 equivalents of TA-G were added relative to PETCP. If 1 equivalent of TA-G was added to PETCP, the viscosity of the solution increased with polymerization, but gelation was not observed (Tables S1 and S2, and Fig. S1). Moreover, partial gelation was observed when 2 equivalents of TA-G were added. Gelation of the entire system was observed when 3 or more equivalents of TA-G were added, and when 5 equivalents of TA-G were added, a transparent and elastic polymer gel was obtained.

To monitor the degree of cross-linking over time of monodisperse PNIPA polymerized with PETCP as an initiator and TA-G as a multifunctional terminating agent, the obtained polymer network was hydrolyzed and the molecular weights of the resultant polymers were measured by SEC. The ester linkage of the initiator is readily hydrolyzed by aqueous alkaline solution, whereas the bond obtained from the addition reaction of PNIPA to TA-G is stable under the same conditions. Thus, the obtained polymer gel was placed in an aqueous alkaline solution and allowed to stand for an appropriate period of time, affording polymers separated from the initiator. In other words, in the obtained polymer network, it is possible to know the structure of PNIPA chains in various combinations with TA-G, as shown in Fig. 4. The SEC results of the polymers obtained by alkaline hydrolysis of both the star polymer and the polymer network synthesized without and with adding TA-G are shown in Fig. 4b, c. The SEC results of the star polymer and its hydrolysate show that there is not much difference in the lengths of the four chains (Fig. 4b). This result indicates that a star polymer consisting of relatively monodisperse four polymer chains was obtained from the first polymerization reaction. On the other hand, the SEC trace shown in Fig. 4c displays several peaks and shoulders, revealing that PNIPA and TA-G underwent an addition reaction but became star polymers with different numbers of bonds. It can be seen that PNIPA is present as a dangling chain that has reacted with TA-G only at one site or not at all. In other words, TA-G has enough functional groups to bind with PNIPA chains having four halogenated ends by the addition reaction, but in this reaction system, the formation of a perfect mesh without dangling chains shown in Scheme 1 was not achieved. The reasons why the crosslinking reaction was not perfect were that the termination reaction between TA-G and the end of the polymer chain was not efficient and that the polymer chain that had reacted with TA-G could not react efficiently with the radical species on other polymer chains. However, a polymer network with narrow MWD polymers between the cross-linking points is obtained and is in a relatively homogeneous state. Furthermore, the network structure is extremely well formed compared with that of a gel 


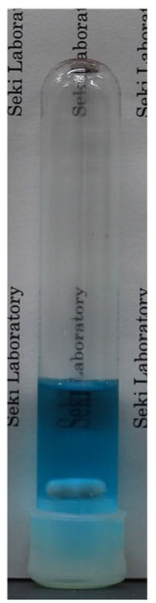

$1 \mathrm{~h}$

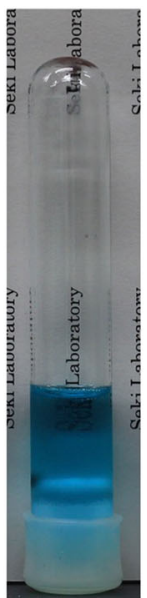

$2 \mathrm{~h}$

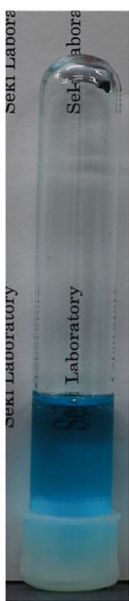

$3 \mathrm{~h}$

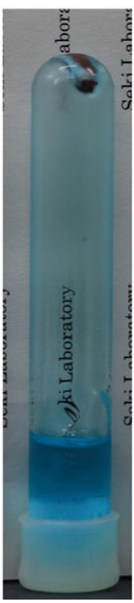

$4 \mathrm{~h}$

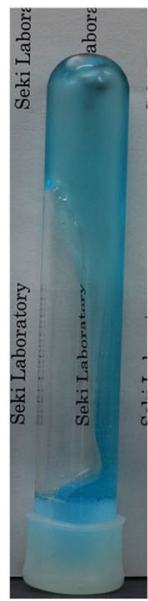

$5 \mathrm{~h}$

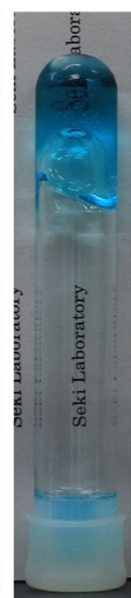

$6 \mathrm{~h}$

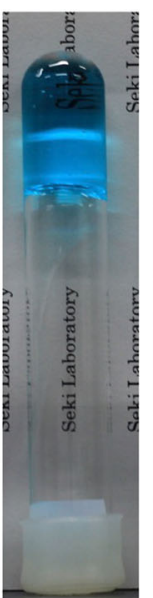

$7 \mathrm{~h}$

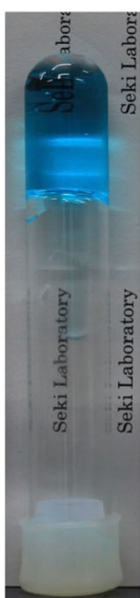

$8 \mathrm{~h}$

Fig. 3 Gelation using the combination of fast-living radical polymerization and the slow radical addition reaction ([NIPA]:[1]:[CuCl]:[Me ${ }_{6}$ TREN]:[TA-G] = 2000:1:20:20:5, [NIPA] $=2 \mathrm{M}, \mathrm{DMF}: \mathrm{H}_{2} \mathrm{O}=25: 75$, temperature $=4^{\circ} \mathrm{C}$, I: initiator)

(a)<smiles>CC(Cl)C(=O)OCC(COC(=O)C(C)Cl)(COC(=O)C(C)Cl)C(=O)O</smiles>

PETCP: degradable 占

(b) tetra-PNIPA

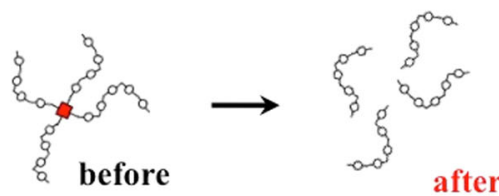

(c) PNIPA gel

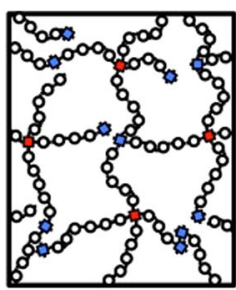

before<smiles>C=CCN1C(=O)N(CC=C)C2C1N(CC=C)C(=O)N2CC=C</smiles>

TA-G: non-degradable 누 
prepared by the conventional method. Even under solvent conditions that allow polymer chains with a narrower MWD to be synthesized, gelation reactions occur due to the addition of metallic copper, facilitating the formation of a more homogeneous and complete network by controlling further reactions (see Supporting Information Figs. S2-S4).

\section{Conclusions}

In this study, a polymerization reaction generated from a four-branched halogenated organic initiator utilizing a copper catalyst was combined with a radical addition reaction between the resultant halogen-terminated polymer and a four-branched allyl compound driven by the same catalyst. As a result, we found a method that can be employed to inherently prepare a polymer network composed of polymer chains of a narrow MWD by simply mixing the necessary compounds. The polymer gels synthesized by this method have a more homogeneous network structure than conventional polymer gels and almost no short-chain branching. Since this reaction can be applied to not only NIPA but also various other vinyl monomers, it can be used to prepare gels having homogeneous polymer network structures composed of various polymers. The resulting polymer gels having homogeneous polymer network structures may exhibit various functional properties based on the network structure composed of polymers of a narrow MWD. Since the best conditions for controlling the molecular weight cannot necessarily be utilized and the reaction with the multifunctional terminating agent is not optimized stoichiometrically, this reaction can be further improved. If we can discover better polymerization and cross-linking reactions, we will be able to prepare a polymer gel with a completely homogeneous network size. Such a polymer gel may be useful as a material with a high molecular sieving ability, a selective concentration ability for specific substances, and space for synthesizing a relatively large substance.

\section{Experimental Materials}

$\mathrm{N}$-Isopropylacrylamide (NIPA) and tris(2-dimethylaminoethyl) amine ( $\left.\mathrm{Me}_{6} \mathrm{TREN}\right)$ were kindly provided by Kohjin and Mitsubishi Chemical. Copper(I) chloride $(\mathrm{CuCl})$, hydrochloric acid $(\mathrm{HCl}), N, N$-dimethylformamide (DMF), toluene, sodium hydroxide $(\mathrm{NaOH})$, and potassium chloride $(\mathrm{KCl})$ were purchased from Kanto Chemical. Hexane, tetrahydrofuran (THF), and methanol were purchased from Kishida Chemistry and used after distillation. Copper powder $(\mathrm{Cu}(0), 75 \mu \mathrm{m})$ and lithium chloride $(\mathrm{LiCl})$ were purchased from Wako Pure Chemical Industries and used without purification. All water used in this study was purified with a Milli-Q Labo system
(MILLIPORE). NIPA was recrystallized twice with a toluene/hexane mixed solvent system, suction filtered, dried, and used. $\mathrm{Me}_{6}$ TREN and DMF were purified by vacuum distillation. $\mathrm{CuCl}$ was dissolved in concentrated $\mathrm{HCl}$ and added dropwise to water, and the obtained precipitate was suction filtered, dried, and used. Pentaerythritol tetra(2-chloropropionate) (PETCP) was purchased from Tokyo Chemical Industry as a special-order product and used as a multifunctional initiator. 1,3,4,6Tetraallylglycoluril (TA-G) was provided by Shikoku Chemicals Corporation and used as a multifunctional terminating agent.

\section{Synthesis of star-branched star PNIPA}

In a flask purged with nitrogen, $\mathrm{CuCl}$ (or $\mathrm{CuCl}$ and $\mathrm{Cu}$ (0)) and $\mathrm{Me}_{6}$ TREN were dissolved in water and stirred to disproportionate the catalyst. After $1 \mathrm{~h}$, a solution of NIPA and PETCP dissolved in DMF/water was added, and the reaction was initiated under an argon atmosphere. During the reaction, the temperature was kept constant, and stirring was continued with a stirrer.

A part of the reaction solution was sampled with a syringe, and ${ }^{1} \mathrm{H}$ NMR analysis was performed. Based on the aldehyde peak $(1 \mathrm{H}, 8.0 \mathrm{ppm})$ of DMF, the amount of which does not change during the reaction, the integral ratio of the proton peak $(1 \mathrm{H}, 5.5 \mathrm{ppm})$ of the vinyl group of NIPA was determined, and Eq. (1) was used to calculate the reaction rate.

$$
\text { Conv } \cdot(\%)=\left(1-\frac{I_{\mathrm{NIPA}_{t}}}{I_{\mathrm{NIPA}_{0}}}\right) \times 100
$$

$I_{\text {NIPA } t}$ integration ratio of the peak derived from the vinyl group of NIPA $(1 \mathrm{H}, 5.5 \mathrm{ppm})$ at reaction time $t$.

The sampled solution was diluted with THF and purified on a silica gel column, and SEC analysis was carried out. After drying the sample purified with a silica gel column, it was diluted with an eluent to a final concentration of $0.5 \mathrm{wt} \%$.

PETCP has four hydrolyzable ester groups, and the resulting star polymer can be decomposed into four-arm polymers. Therefore, the obtained four-branched star PNIPA was purified and hydrolyzed, and SEC analysis was carried out. The solution sampled during the latter stage of the reaction was diluted with THF; the catalyst was removed by a silica gel column, and the resulting solution was added to a dialysis tube (purchased from Spectrum Laboratories Inc., molecular weight cutoff $=1000$ ). The solution was dialyzed against methanol for 2 days and then against ion-exchanged water for 5 days, and the purified product was obtained by lyophilization. The purified product was dissolved in an aqueous solution of $0.2 \mathrm{M} \mathrm{KCl}$ and $0.2 \mathrm{M} \mathrm{NaOH}$ and hydrolyzed by stirring for 3 days. After neutralization with dilute $\mathrm{HCl}$, the salts were again removed by dialysis against deionized water for 3 days, and 
freeze-drying was carried out. The obtained purified product was diluted with an eluent to a final concentration of $0.5 \mathrm{wt} \%$, and SEC analysis was performed.

\section{Preparation of the PNIPA gel}

$\mathrm{CuCl}$ (or $\mathrm{CuCl}$ and $\mathrm{Cu}(0)$ ) and $\mathrm{Me}_{6}$ TREN were dissolved in water in a nitrogen-purged test tube and stirred to disproportionate the catalyst. The total volume at this time was $0.5 \mathrm{ml}$. After $1 \mathrm{~h}$, a DMF/water monomer solution in which NIPA, PETCP, and TA-G were dissolved was injected into the catalyst solution, and the reaction was initiated under an argon atmosphere. The total volume of the reaction solution was $4.0 \mathrm{ml}$. The monomer concentration was set to 2 $\mathrm{M}$, which was considered equal to or higher than the overlapping concentration of the obtained PNIPA. After adding the monomer solution, stirring was continued with a stirrer for $10 \mathrm{~min}$, and the temperature of the solution was kept constant. Because of the heat generated by the polymerization, the reaction mixture was stirred with sufficient care so that it could be gradually and efficiently cooled. Stirring was stopped; the reaction temperature was kept constant, and the mixture was allowed to stand. Finally, changes in the reaction solution were observed.

\section{Hydrolysis of the PNIPA gel}

PETCP has four ester groups that can be hydrolyzed by aqueous alkaline solution, whereas the structure of TA-G is not hydrolyzed under the same conditions. That is, selective hydrolysis of only the cross-linking points in the network structure of the obtained PNIPA gel derived from the initiator can be achieved. Therefore, the obtained PNIPA gel was hydrolyzed, and SEC measurements were carried out to obtain information on the cross-linked structure formed by TA-G. The experimental steps are described below. The obtained PNIPA gel was thoroughly washed with ion-exchanged water for 5 days. The washed PNIPA gel was immersed in an aqueous solution of $0.2 \mathrm{M} \mathrm{KCl}$ and $0.2 \mathrm{M} \mathrm{NaOH}$ and hydrolyzed by stirring for 3 days. After neutralizing the hydrolysate with dilute aqueous $\mathrm{HCl}$ solution, the salts were removed by dialyzing the resultant solution in a dialysis tube (purchased from Spectrum Laboratories Inc., molecular weight cutoff $=1000$ ) for 3 days against ion-exchanged water. The purified product obtained was diluted with THF, and the remaining catalyst was removed by a silica gel column. Next, if the sample was not soluble in THF, a very small amount of DMF was added to dissolve it. After drying the solution obtained from column purification, it was diluted with an eluent to a final concentration of $0.5 \mathrm{wt} \%$, and SEC analysis was carried out.

\section{Measurements \\ Proton nuclear magnetic resonance spectrum ( ${ }^{1} \mathrm{H}$ NMR)}

For analytical equipment, a JNM-A400 spectrometer (JEOL) was used. Acetone- $\mathrm{d}_{6}$ (containing $0.03 \%$ TMS) purchased from Kanto Chemical Co., Ltd. was used as the deuterated solvent.

\section{Size-exclusion chromatography (SEC)}

Tosoh $\alpha-M$ and $\alpha-3000$ columns were connected to each other. A DMF solution in which $0.1 \mathrm{M} \mathrm{LiCl}$ was dissolved was used as the eluent. For analytical equipment, a PU-2080 pump and an RI-2031 detector (JASCO Corporation) were used. The flow rate was $1.0 \mathrm{ml} / \mathrm{min}$, and the temperature was $40^{\circ} \mathrm{C}$. Poly(methyl-methacrylate) (PMMA) was used as a standard sample, and PMMA of various molecular weights was purchased from Varian $\left(M_{\mathrm{p}}: 645-1,677,000 ; M_{\mathrm{w}} / M_{\mathrm{n}}: 1.02-1.29\right)$ and Aldrich $\left(M_{\mathrm{p}}: 2,730,000 ; M_{\mathrm{w}} / M_{\mathrm{n}}: 1.25\right)$.

\section{Acknowledgements}

$\mathrm{N}$-Isopropylacrylamide and 1,3,4,6-tetraallylglycoluril were kindly provided by Kohjin (Tokyo, Japan) and Shikoku Chemicals Corporation, respectively.

\section{Author contributions}

Y.T. designed the project. Y.J. performed the experiments. All authors discussed the results and contributed to the data interpretation. Y.T. and K.S. wrote the manuscript

\section{Conflict of interest}

The authors declare that they have no conflict of interest.

\section{Publisher's note}

Springer Nature remains neutral with regard to jurisdictional claims in published maps and institutional affiliations.

Supplementary information is available for this paper at https://doi.org/ 10.1038/s41427-018-0074-X.

Received: 9 May 2018 Revised: 2 July 2018 Accepted: 3 July 2018.

Published online: 30 August 2018

\section{References}

1. Osada, Y. \& Gong, J. P. Soft and wet materials: polymer gels. Adv. Mater. $\mathbf{1 0}$ 827 (1998).

2. Tanaka, T. Gels. Sci. Am. 244, 124 (1981).

3. Imran, A. B. et al. Extremely stretchable thermosensitive hydrogels prepared by introducing polyrotaxane-based slide-ring cross-linkers and ionic groups into the polymer network. Nat. Commun. 5, 5124 (2014).

4. Alvarez-Lorenzo, T. et al. Polymer gels that memorize elements of molecular conformation. Macromolecules 33, 8693 (2000).

5. Takeoka, Y. et al. First order phase transition and evidence for frustrations in polyampholytic gels. Phys. Rev. Lett. 82, 4863 (1999).

6. Takeoka, Y. \& Seki, T. Visualizing conformations of subchains by creating optical wavelength-sized periodically ordered structure in hydrogel. Langmuir 22, 10223 (2006)

7. Nakayama, D., Takeoka, Y., Watanabe, M. \& Kataoka, K. Simple and precise preparation of a porous gel for a colorimetric glucose sensor by a templating technique. Angew. Chem. Int. Ed. 42, 4197 (2003).

8. Matsubara, K., Watanabe, M. \& Takeoka, Y. A thermally adjustable multicolor photochromic hydrogel. Angew. Chem. Int. Ed. 46, 1688 (2007).

9. Shibayama, M. Small-angle neutron scattering on polymer gels: phase behavior, inhomogeneities and deformation mechanisms. Polym. J. 43, 18 (2011).

10. Bromberg, L., Grosberg, A. Y., Matsuo, E. S., Suzuki, Y. \& Tanaka, T. Dependency of swelling on the length of subchain in poly(N,N-dimethylacrylamide)-based gels. J. Chem. Phys. 106, 2906 (1997) 
11. Bastide, J., Leibler, L. \& Prost, J. Scattering by deformed swollen gels - butterfly isointensity patterns. Macromolecules 23, 1821 (1990).

12. Urayama, K. \& Kohjiya, S. Crossover of the concentration dependence of swelling and elastic properties for polysiloxane networks crosslinked in solution. J. Chem. Phys. 104, 3352 (1996).

13. Urayama, K., Kawamura, T. \& Kohjiya, S. Elastic modulus and equilibrium swelling of networks crosslinked by end-linking oligodimethylsiloxane at solution state. J. Chem. Phys. 105, 4833 (1996)

14. Ida, S., Kimura, R., Tanimoto, S. \& Hirokawa, Y. End-crosslinking of controlled telechelic poly (N-isopropylacrylamide) toward a homogeneous gel network with photo-induced self-healing. Polym. J. 49, 237 (2017).

15. Sakai, T. et al. Design and fabrication of a high-strength hydrogel with ideally homogeneous network structure from tetrahedron-like macromonomers. Macromolecules 41, 5379 (2008).

16. Matsumoto, A. et al. Demonstration of thermo-sensitive tetra-gel with implication for facile and versatile platform for a new class of smart gels. J. Biomater. Sci. Polym. Ed. 28, 1000 (2017).

17. Wang, J. et al. Preparation of well-defined propargyl-terminated tetra-arm poly (N-isopropylacrylamide)s and their click hydrogels crosslinked with _-cyclodextrin. Polymers 8, 93 (2016).

18. Kato, M., Kamigaito, M., Sawamoto, M. \& Higashimura, T. Polymerization of methyl-methacrylate with the carbon-tetrachloride dichlorotris(triphenylphosphine)ruthenium(li) methylaluminum bis(2,6-Di-tert-butylphenoxide) initiating system - possibility of living radical polymerization. Macromolecules $\mathbf{2 8}$ 1721 (1995).

19. Rizzardo, E. et al. RAFT polymerization: adding to the picture. Macromol. Symp. 248, 104 (2007).

20. Hawker, C. J. Molecular-weight control by a living free-radical polymerization process. J. Am. Chem. Soc. 116, 11185 (1994).

21. Nguyen, N. H., Rosen, B. M. \& Percec, V. SET-LRP of N,N-dimethylacrylamide and of $\mathrm{N}$-isopropylacrylamide at 25 degrees $\mathrm{C}$ in protic and in dipolar aprotic solvents. J. Polym. Sci. Pol. Chem. 48, 1752 (2010).
22. Masci, G., Giacomelli, L. \& Crescenzi, V. Atom transfer radical polymerization of $\mathrm{N}$-isopropylacrylamide. Macromol. Rapid Commun. 25, 559 (2004)

23. Millard, P. E., Mougin, N. C., Boker, A. \& Muller, A. H. E. Controlling the fast ATRP of N-isopropylacrylamide in water. ACS Sym Ser. 1023, 127 (2009).

24. Mizutani, M., Satoh, K. \& Kamigaito, M. Metal-catalyzed simultaneous chainand step-growth radical polymerization: marriage of vinyl polymers and polyesters. J. Am. Chem. Soc. 132, 7498 (2010).

25. Mizuntani, M., Satoh, K. \& Kamigaito, M. Degradable Poly(N-isopropylacrylamide) with tunable thermosensitivity by simultaneous chain- and step-growth radical polymerization. Macromolecules 44, 2382 (2011).

26. Rosen, B. M. \& Percec, V. Single-electron transfer and single-electron transfer degenerative chain transfer living radical polymerization. Chem. Rev. 109, 5069 (2009).

27. Amiya, T., Hirokawa, Y., Hirose, Y., Li, Y. \& Tanaka, T. Reentrant phase-transition of N-isopropylacrylamide gels in mixed-solvents. J. Chem. Phys. 86, 2375 (1987).

28. Jung, S. C., Oh, S. Y. \& Bae, Y. C. Reentrant swelling behavior of thermosensitive N-isopropylacrylamide nano-sized gel particles. Polymer 50, 3370 (2009).

29. Kojima, H. \& Tanaka, F. Reentrant volume phase transition of cross-linked poly (N-isopropylacrylamide) gels in mixed solvents of water/methanol. Soft Matter 8, 3010 (2012).

30. Costa, R. O. R. \& Freitas, R. F. S. Phase behavior of poly(N-isopropylacrylamide) in binary aqueous solutions. Polymer 43, 5879 (2002).

31. Hirokawa, Y. et al. Sponge-like heterogeneous gels: hierarchical structures in Poly(N-isopropylacrylamide) chemical gels as observed by combined scattering and confocal microscopy method. Macromolecules 41, 8210 (2008).

32. Halperin, A., Kroger, M. \& Winnik, F. M. Poly(N-isopropylacrylamide) phase diagrams: fifty years of research. Angew. Chem. Int. Ed. 54, 15342 (2015) 